\title{
Experimental studies of phase conjugation with depleted pumps in photorefractive media
}

\author{
Sze-Keung Kwong, Young-Hoon Chung, Mark Cronin-Golomb, ${ }^{*}$ and Amnon Yariv \\ California Institute of Technology, Pasadena, California 91125
}

Received January 28, 1985; accepted March 29, 1985

\begin{abstract}
We describe the experimental measurement of phase-conjugate reflectivity versus various ratios of input-beam intensities in photorefractive barium titanate and strontium barium niobate crystals. The experimental results are compared with the theoretical prediction from the coupled-wave theory. Three different methods to measure the nonlinear coupling constant of the crystal are also presented and compared.
\end{abstract}

Many novel optical devices have been made possible by photorefractive nonlinearities in highly electro-optic crystals, such as barium titanate and strontium barium niobate. These include passive (self-pumped) phase conjugators, ${ }^{1-3}$ ring resonators, ${ }^{4}$ interferometers,,${ }^{5}$ and optical bistable devices. ${ }^{6}$ The design and interpretation of these devices have been facilitated by solutions of the nonlinear coupled-wave equations of the photorefractive effect. Solutions of the two-beam-coupling equations have been known and experimentally studied for some time now. ${ }^{7-9}$ However, the four-beam equations of phase conjugation have been solved only recently, ${ }^{10-12}$ and there is little experimental evidence ${ }^{13}$ to verify this theory apart from qualitative agreement with observed device behavior.

Here we present some experimental studies of fourwave mixing in both photorefractive barium titanate $\left(\mathrm{BaTiO}_{3}\right)$ and strontium barium niobate $\left[\mathrm{Sr}_{0.6} \mathrm{Ba}_{0.4^{-}}\right.$ $\left.\mathrm{Nb}_{2} \mathrm{O}_{6},(\mathrm{SBN})\right]$ crystals. The experimental arrangement is shown in Fig. 1. The output of the argon-ion laser at $514 \mathrm{~nm}$ was expanded and spatial filtered into a $\sim 2.5-\mathrm{cm}$-diameter beam. The total beam intensity $I_{1}+I_{2}+I_{4}$ was $0.3 \mathrm{~W} / \mathrm{cm}^{2}$. The crystal, $\mathrm{C}$, was either a $4.5 \mathrm{~mm} \times 4.5 \mathrm{~mm} \times 5 \mathrm{~mm} \mathrm{BaTiO}$ or a $5 \mathrm{~mm} \times 5 \mathrm{~mm}$ $\times 6 \mathrm{~mm}$ SBN crystal. Both crystals were electrically poled into a single domain before the experiment. Since only a small part $(\sim 5 \mathrm{~mm} \times 5 \mathrm{~mm})$ of the central portion of the expanded beam was used, we can approximate all the incoming beams to the crystal as plane waves. The half-wave plate and the polarizing beam splitter combination forms a lossless beam splitter with a transmission and reflection ratio that can be varied simply by rotating the half-wave plate. In order to make sure that only one grating (the transmission grating) dominated the system, the optical path lengths of beams 1 and 4 were adjusted to be the same, while the optical path difference between beams 2 and 4 was adjusted to be much longer than the coherence length of the light source $(<1 \mathrm{~cm})$. The angle between beams 1 and 4 was made small $\left(10^{\circ}\right)$ to increase the interaction length. The orientation of the crystals with respect to the input beams is shown in Fig. 2, where $\theta_{1}$ and $\theta_{2}$ are the angles of beams 1 and 4, respectively, with respect to the normal of the crystal C. All the beams were polarized in the plane of Fig. 1 to utilize the larger $r_{42}$ electro-optical coefficient of $\mathrm{BaTiO}_{3}$. The phaseconjugate beam $I_{3}$ was separated from beam 4 by a beam splitter $\mathrm{BS}$ and was detected by detector $\mathrm{D}_{3}$. Part of the intensities of beams 1,2 , and 4 were reflected at the surface of the crystal and were detected by detectors $\mathrm{D}_{1}, \mathrm{D}_{2}$, and $\mathrm{D}_{4}$, respectively. All the data collected by detectors $D_{1}-D_{4}$ were sent to a minicomputer for data analysis.

The experimental results for the $\mathrm{BaTiO}_{3}$ crystal are shown in Fig. 3. The phase-conjugate reflectivity, $R=$ $I_{3}(0) / I_{4}(0)$, was plotted against the probe ratio, $q=$ $I_{4}(0) /\left[I_{1}(0)+I_{2}(l)\right]$, at various pump ratios, $r=I_{2}(l) /$ $I_{1}(0)$. A set of theoretical curves was also plotted.12 These theoretical curves give a best fit to the experimental curves if the nonlinear coupling constant $\dot{\gamma} l$ is chosen to be $-0.9 .^{14}$ Similar experiments were also done on SBN, whose large electro-optic coefficient is $r_{33}$. The results are shown in Fig. 4. The coupling constant chosen from the best curve fitting is -1.3 . This pro-

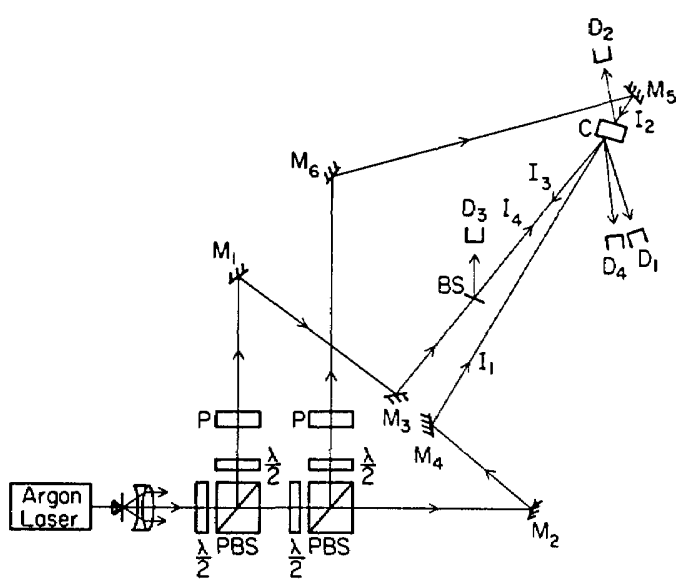

Fig. 1. Experimental configuration for measuring phaseconjugate reflectivity versus various beam ratios. The elements are a $10 \times$ objective, a pinhole, and a lens; $\lambda / 2$, half-wave plate; PBS's, polarizing beam splitters; P's, polarizers; BS, beam splitter; $C$, crystal; $M_{1}-M_{6}$, mirrors; $D_{1}-D_{4}$, detectors. 


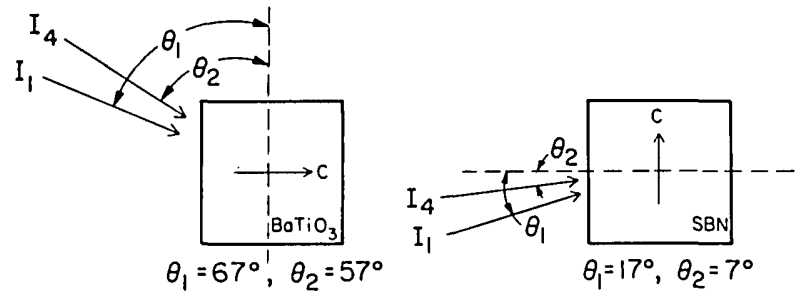

Fig. 2. The orientation of the $\mathrm{BaTiO}_{3}$ and the SBN crystals with respect to the input beams.
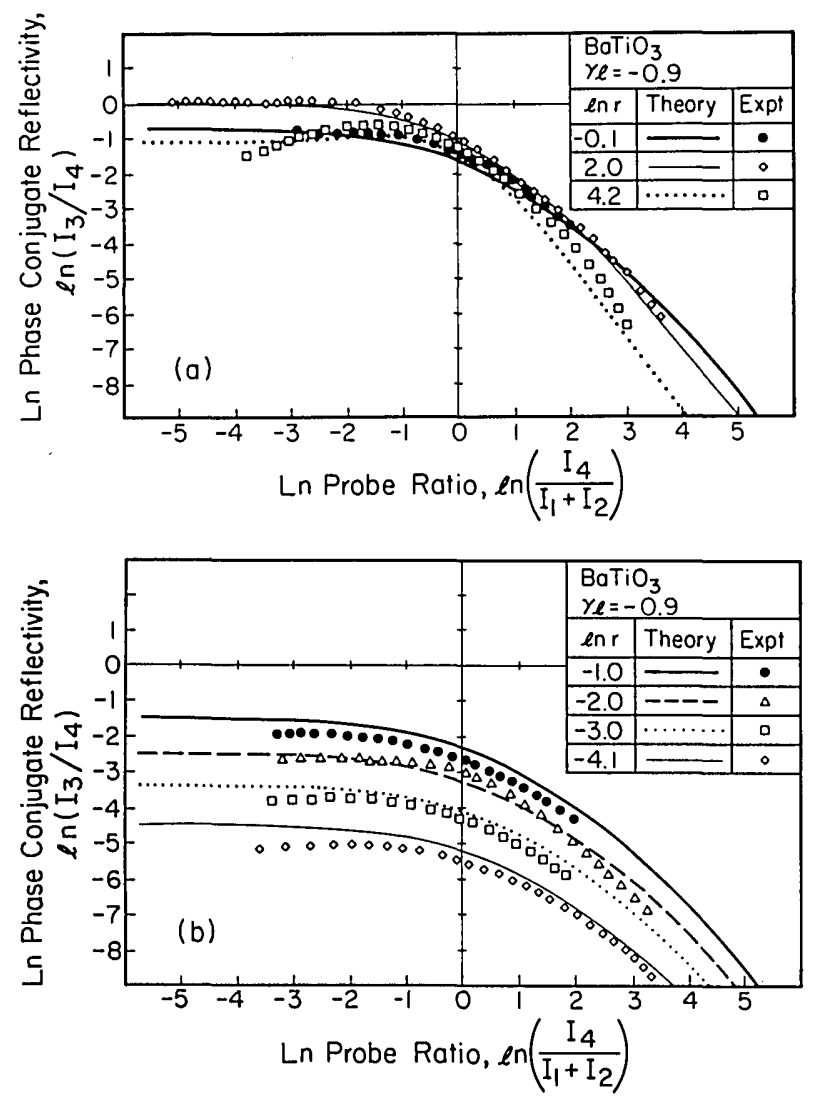

Fig. 3. Experimental and theoretical curves of phase-conjugate reflectivity in $\mathrm{BaTiO}_{3}$ versus probe ratio at natural-log pump ratio: (a) $4.2,2.0,-0.1$, (b) $-1.0,-2.0,-3.0,-4.1$.

vides the first method of determining the nonlinear coupling constant.

We also measured the phase-conjugate reflectivity versus the pump ratio at very small probe ratios (i.e., an undepleted pump approximation, which is $I_{1}, I_{2}, \gg I_{4}$ ), as shown in Figs. 5 and 6 . The experimental curve fits well with the theoretical curve, except at very large and very small pump ratios. From the theory of undepleted pump approximation, ${ }^{10}$ the phase-conjugate reflectivity $R$ is

$$
R=\left|\frac{\sinh \left(\frac{\dot{\gamma} l}{2 \cos \theta_{2}}\right)}{\cosh \left(\frac{\dot{\gamma} l}{2 \cos \theta_{2}}+\frac{\ln r}{2}\right)}\right|^{2} .
$$

Therefore, at the maximum reflectivity,

$$
\dot{\gamma} l=-\cos \theta_{2} \ln r .
$$

From the experimental data, the nonlinear coupling constant ranges from -0.82 to -1.0 for $\mathrm{BaTiO}_{3}$ and from -1.1 to -1.5 for $\mathrm{SBN}$. These values are consistent with the previous values of coupling constants determined by curve fitting.

Finally, we performed a two-wave mixing experiment to measure the coupling constant directly. The formula for the coupling constant is ${ }^{15}$

$$
\dot{\gamma} l=-\frac{1}{2}\left[\cos \theta_{1} \ln \frac{I_{1}(l)}{I_{1}(0)}+\cos \theta_{2} \ln \frac{I_{4}(0)}{I_{4}(l)}\right] .
$$

The experimental arrangement was similar to that shown in Fig. 2, with beam 2 being blocked and detectors $\mathrm{D}_{2}$ and $\mathrm{D}_{3}$ repositioned to measure the transmitted beams $I_{1}(l)$ and $I_{4}(l)$ through the crystal. An experimental plot of coupling constant $\dot{\gamma} l$ against beam ratio $I_{4}(0) / I_{1}(0)$ is shown in Fig. 7. From the prediction of the theory of the photorefractive effect, ${ }^{8,9}$ the coupling constant should be independent of the beam ratio. The slight variation of coupling constant with input-beam ratio, which was also observed by Kuktarev et $a l .,{ }^{8}$ is under investigation. The variation of coupling constant is between -0.8 and -1.3 for $\mathrm{BaTiO}_{3}$ and -0.4 to -1.0

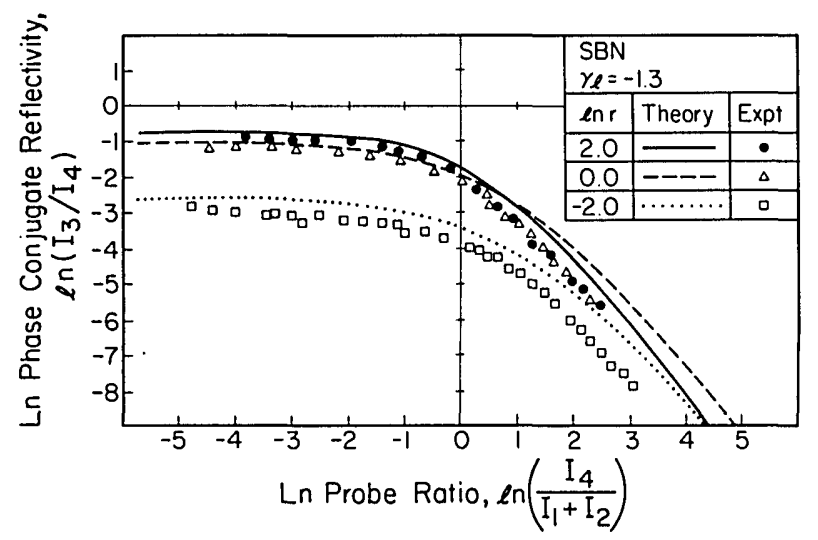

Fig. 4. Experimental and theoretical curves of phase-conjugate reflectivity in SBN versus probe ratio at natural-log pump ratio: $2.0,0.0,-2.0$.

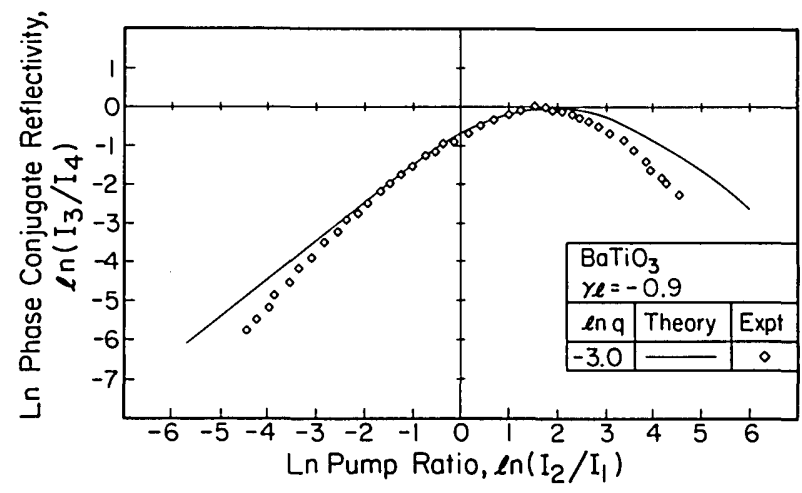

Fig. 5. Experimental and theoretical curves of phase-conjugate reflectivity versus pump ratio in undepleted pump region for $\mathrm{BaTiO}_{3}$. 


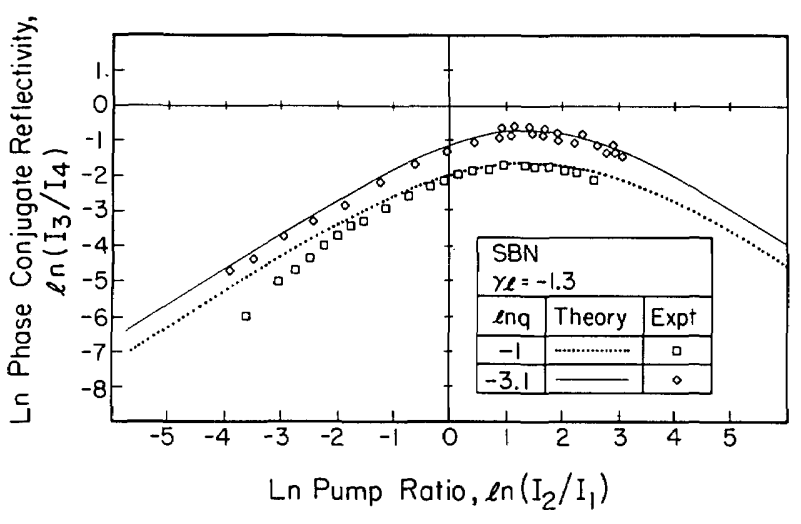

Fig. 6. Experimental and theoretical curves of phase-conjugate reflectivity versus pump ratio in undepleted pump region for SBN.

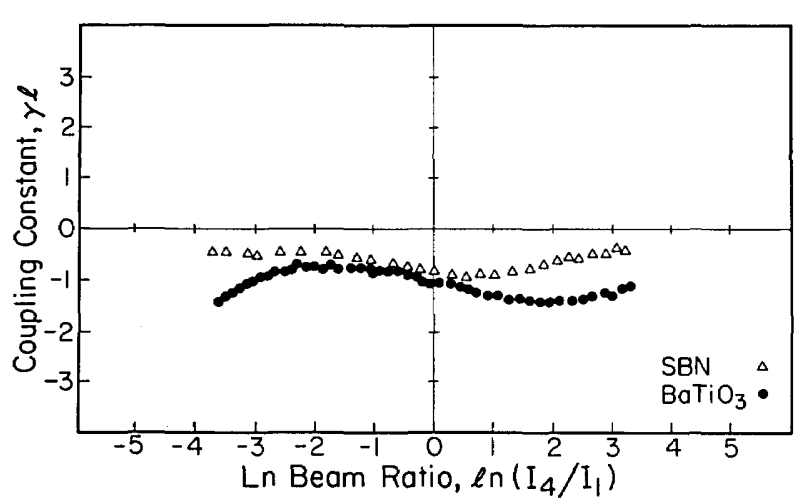

Fig. 7. Two-beam-coupling constant versus ratio of the intensities of the two input beams.

for SBN over the range of beam ratio from 0.02 to 20 . These values are quite consistent with the values obtained by the previous two methods.

In deriving the coupled-wave theory, ${ }^{10-12}$ the $a b$ sorption of the crystal is neglected. Besides absorption, the crystals also give rise to asymmetric scattering (fanning). ${ }^{16,17}$ These combining effects tend to lower the phase-conjugate reflectivity. ${ }^{18}$ The theory also neglects multiple Fresnel reflections within the crystal. Therefore a certain amount of discrepancy between the theory and experiment is expected.

In conclusion, we have verified the coupled-wave theory experimentally by studying the phase-conjugate reflectivity versus various input-beam ratios. Also, we have demonstrated three different methods to deter- mine the nonlinear coupling constant of a crystal at a given orientation.

The authors wish to thank R. Neurgaonkar of Rockwell International for supplying several samples of SBN. This research was supported by the U.S. Air Force Office of Scientific Research, and the U.S. Army Research Office, Durham, North Carolina.

*Present address, Ortel Corporation, 2015 West Chestnut Street, Alhambra, California 91308.

\section{References}

1. M. Cronin-Golomb, B. Fischer, J. O. White, and A. Yariv, Appl. Phys. Lett. 41, 689 (1982).

2. M. Cronin-Golomb, S. K. Kwong, and A. Yariv, Appl. Phys. Lett. 44, 727 (1984).

3. K. R. MacDonald and J. Feinberg, J. Opt. Soc. Am. 73, 548 (1983).

4. J. O. White, M. Cronin-Golomb, B. Fischer, and A. Yariv, Appl. Phys. Lett. 40, 450 (1982).

5. S. K. Kwong, A. Yariv, M. Cronin-Golomb, and I. Ury (to be published).

6. S. K. Kwong, M. Cronin-Golomb, and A. Yariv, Appl. Phys. Lett. 45, 1016 (1984)

7. D. W. Vahey, J. Appl. Phys. 46, 3510 (1975).

8. N. V. Kukhtarev, V. B. Markov, S. G. Odulov, M. S. Soskin, and V. L. Vinetskii, Ferroelectrics 22, 949 (1979).

9. J. Feinberg, D. Heiman, A. R. Tanguay, and R. W. Hellwarth, J. Appl. Phys. 51, 1297 (1980).

10. B. Fischer, M. Cronin-Golomb, J. O. White, and A. Yariv, Opt. Lett. 6, 519 (1981).

11. M. Cronin-Golomb, J. O. White, B. Fischer, and A. Yariv, Opt. Lett. 7, 313 (1982).

12. The theoretical curves were based on the solutions to the coupled-wave equations by M. Cronin-Golomb, B. Fischer, J. O. White, and A. Yariv, IEEE J. Quantum Electron. QE-20, 12 (1984), where we approximated $\theta \simeq$ $\left(\theta_{1}+\theta_{2}\right) / 2$ in plotting these curves. This approximation will not give any significant changes in the curves since $\left(\theta_{1}-\theta_{2}\right)$ is only $10^{\circ}$.

13. J. O. White, Ph.D. dissertation (California Institute of Technology, Pasadena, Calif., 1984, unpublished).

14. The coupling constant $\dot{\gamma} l$ defined in this Letter does not include a geometrical factor $\cos \theta$, whereas this factor was included in the definition of the coupling constant in Refs. $10-12$.

15. The crystal absorption $\alpha$ was already taken into account in deriving this formula.

16. V. V. Voronov, I. R. Dorosh, Yu. S. Kuz'minov, and N. V. Tkachenko, Sov. J. Quantum Electron. 10, 1346 (1980).

17. J. Feinberg, J. Opt. Soc. Am. 72, 46 (1982).

18. In the undepleted pump approximation, we can theoretically calculate the exact amount of reflectivity lowered by the presence of crystal absorption; see Ref. 12 . 